\title{
Embedding Overlap Priors in Variational Left Ventricle Tracking
}

\author{
Ismail Ben Ayed*, Member, IEEE, Shuo Li, and Ian Ross
}

\begin{abstract}
This study investigates overlap priors for variational tracking of the Left Ventricle (LV) in cardiac Magnetic Resonance (MR) sequences. The method consists of evolving two curves toward the LV endo- and epicardium boundaries. We derive the curve evolution equations by minimizing two functionals each containing an original overlap prior constraint. The latter measures the conformity of the overlap between the nonparametric (kernel-based) intensity distributions within the three target regions- $\mathbf{L V}$ cavity, myocardium and background-to a prior learned from a given segmentation of the first frame. The Bhattacharyya coefficient is used as an overlap measure. Different from existing intensity-driven constraints, the proposed priors do not assume implicitly that the overlap between the intensity distributions within different regions has to be minimal. This prevents both the papillary muscles from being included erroneously in the myocardium and the curves from spilling into the background. Although neither geometric training nor preprocessing were used, quantitative evaluation of the similarities between automatic and independent manual segmentations showed that the proposed method yields a competitive score in comparison with existing methods. This allows more flexibility in clinical use because our solution is based only on the current intensity data, and consequently, the results are not bounded to the characteristics, variability, and mathematical description of a finite training set. We also demonstrate experimentally that the overlap measures are approximately constant over a cardiac sequence, which allows to learn the overlap priors from a single frame.
\end{abstract}

Index Terms - Left Ventricle tracking, cardiac Magnetic Resonance Images (cardiac MRI), variational image segmentation, overlap priors, active contours, level sets.

\section{INTRODUCTION}

Accurate tracking of the Left Ventricle (LV) endo- and epicardium boundaries in 2D cardiac Magnetic Resonance (MR) sequences is needed for the analysis and quantification of the regional motion of the LV [44]. It plays an essential role in the diagnosis of cardiovascular diseases related to localized regions with movement abnormalities. The standardized myocardial segmentation [43] suggests selecting representative 2D cardiac slices to generate 17 standardized LV segments over which we can assess the regional wall motion abnormalities. This standardization is commonly used for regional analysis of the LV function. Such analysis requires accurate LV

Copyright (c) 2009 IEEE. Personal use of this material is permitted. However, permission to use this material for any other purposes must be obtained from the IEEE by sending a request to pubs-permissions@ieee.org.

Manuscript received January 8, 2009; revised April 21, 2009. The Associate Editor responsible for coordinating the review of this paper and recommending its publication was A. Amini. Asterisk indicates corresponding author.

I. Ben Ayed and S. Li are with General Electric Canada (GE Healthcare), London, ON, Canada (e-mail:ismail.benayed@ge.com)

I. Ross is with the London Health Sciences Centre, London, ON, Canada. tracking, which consists of segmenting each frame into three target regions (objects): LV cavity, myocardium and background (refer to Fig. 3). Manual labeling is time-consuming. Therefore, an automatic tracking is desired. Although an impressive research effort has been devoted to this task [1][9], [15]-[32], existing methods are still insufficiently flexible for routine clinical use. Accurate LV tracking is acknowledged as a difficult problem because of several reasons:

(a) A significant overlap exists between the intensity distributions within the cardiac regions (cf. the typical example in Fig. 3)

- The myocardium and the papillary muscles inside the cavity are connected and have almost the same intensity (refer to Fig. 3 e). In this connection, several studies have addressed specifically the problems with the papillary muscles [9], [8].

- The LV cavity has an intensity distribution similar to the right ventricle [25] (refer to Fig. 3 d).

(b) There are no boundaries between the epicardium and some cardiac regions [25].

(c) The substantial variations in size, shape and intensity between subjects, particularly those with pathological patterns, makes geometric/photometric models hard to build from a finite training set [7], [29].

Most of existing approaches to the LV tracking compute a pixelwise correspondence between the current image (or frame) and model distributions of photometric (intensitybased) and geometric ${ }^{1}$ properties of the target objects. Model distributions are generally learned from a training set, i.e., a finite set of hand-labeled images, and embedded in the segmentation via two standard frameworks: variational active contours/level-sets [34] (such as [1]-[3], [15]-[25]) and active appearance/shape models [33] (such as [28]-[32]). In the variational framework, the problem is commonly stated as the minimization of a functional containing two constraints: a geometric constraint which biases the solution toward a specific geometric knowledge learned from a finite training set and an intensity-driven constraint based on the Maximum Likelihood (ML) principle [34]. The latter maximizes the conditional probability of pixel intensity given the assumed model distribution within each region. Unfortunately, a ML intensity-driven constraint is sensitive to inaccuracies in estimating model distributions [10]. More importantly, it can not incorporate information about the overlap between the intensity distributions within different regions. Based on the evaluation of a pixelwise correspondence between the image

\footnotetext{
${ }^{1}$ Geometric properties include object shape, spatial position, and interobject spatial relations.
} 
and the models, ML intensity-driven constraints assume implicitly that the distribution overlap within different regions has to be minimal. Such assumption is often violated in medical images. For instance, the pixelwise information is misleading in the case of the LV due to the "significant" (cf. the typical example in Fig. 3) overlap between the distributions within different cardiac regions. Consequently, the use of trainingbased geometric constraints in conjunction with ML intensitydriven constraints was inevitable to obtain satisfying results [15]-[23]. Similar to variational level-set approaches, active appearance/shape models compute a pixelwise correspondence between the image and the models [40]. As we will show in the experiments (section IV), embedding global information about the overlap between the intensity distributions within the segmentation regions is important. In the current study, we embed overlap priors in variational image segmentation and demonstrate the effectiveness and flexibility of the proposed method in the context of the LV tracking.

We state the tracking of the endo- and epicardium boundaries in a cardiac MR sequence as the evolution of two active curves. We derive the curve evolution equations by minimizing two functionals each containing an original overlap prior. The latter measures the conformity of the overlap between the nonparametric (kernel-based) intensity distributions within the three target regions-LV cavity, myocardium and backgroundto a prior learned from a given segmentation of the first frame. The Bhattacharyya coefficient is used as an overlap measure. The contributions of this study are not only in the application context but also in the scope of general-purpose segmentation.

\section{A. The contributions of this study}

1) The LV tracking context: In the application context, the contributions of this study can be summarized as follows.

(a) Using only the current intensity data, i.e., even without a geometric training, the proposed method still yields a competitive segmentation score because the overlap priors prevent both the papillary muscles from being included erroneously in the myocardium and the curves from spilling into the background. This allows more flexibility in clinical use, particularly when dealing with abnormal subjects where the left ventricle undergoes high and unseen (unexpected) geometric variations. The overlap priors lead to a LV tracking which has several advantages over existing methods:

- The results are not bounded to some geometric/photometric properties and variations learned from finite training set.

- The proposed method is not prone to the practical difficulties related to the choice of training set and the corresponding mathematical models and ad hoc parameters.

- With the use of overlap priors, systematic bias caused in some cases by geometric-training constraints [7] can be relaxed or weakened, particularly with abnormal subjects where the left ventricle undergoes high and unseen (unexpected) geometric variations. It is worth mentioning, however, that the proposed overlap prior is not an alternative to geometric priors. Geometric and overlap constraints are orthogonal and can be used along with each other. The overlap prior is rather an alternative to existing intensity constraints that are unlikely to yield acceptable results without a geometric training. It can be used in conjunction with geometric constraints in medical applications where a reliable geometric information can be learned from a training set.

- No assumption is made as to the parametric distributions of photometric/geometric data because the current study uses a nonparametric description.

- Explicit curve coupling [3], [25] is not required because the proposed functionals and two-step minimization yield an implicit coupling.

(b) We also demonstrate experimentally that the used overlap measures are approximately constant over a cardiac sequence. This result is important because it allows to learn the overlap priors from a single frame of the current data. It can be also the basis of future investigations of the LV segmentation/tracking.

2) The general-purpose segmentation context: The current study is most related, but not similar, to the recent segmentation/tracking investigations [10]-[14] which have shown the advantages of using distribution metrics over the ML principle. In this connection, there is a fundamental difference between the proposed method and the methods in [10]-[14]. Similar to the ML principle, the methods in [10]-[14] are based on the following implicit assumption: The overlap between the distributions of intensity data within different regions in the current image/frame has to be minimal. Unfortunately, such assumption may not be valid in cardiac imagery as well as many other applications. Although those methods have been effective in some cases, they are not versatile enough to deal with situations in which a "significant" (cf. the left ventricle example in Fig. 3) overlap exists between the distributions within different regions. The proposed method relaxes the assumption of minimal overlap. It can be viewed as a generalization of [10]-[14] for situations in which an overlap exists between the distributions within different regions and, consequently, it is more widely applicable than existing general-purpose methods. As we will show in the context of the LV tracking (section IV), embedding information about such overlap in the tracking functional would be very useful.

The remainder of this paper is organized as follows. The next section gives a brief review, focusing on general-purpose variational methods as well as the LV tracking. Section III contains the theoretical contribution: after introducing the appropriate notations and defining the proposed functionals, we derive the descent minimization equations via curve evolution. Section IV describes a quantitative performance evaluation of the proposed method over ten datasets by comparisons with manual segmentations and other variational methods. It also demonstrates the advantage of using overlap constraints over existing intensity constraints, reports the statistics the overlap priors/measures, and finally depicts a representative sample of the results. Section V contains a discussion.

\section{Previous WORK}

\section{A. General-purpose variational segmentation}

The variational level-set segmentation framework, which uses active curves to delineate the target objects, has been gen- 
erally effective and flexible [10]-[14], [34]-[38]. It has become very popular in computer vision and medical image analysis for several reasons: (a) the solution is sought following the optimization of a cost functional which balances the influence of image data and prior knowledge in a flexible, principled, and transparent way. This applies to a wide range of functionals including intensity-driven, prior-knowledge and applicationspecific functionals; (b) the level-set representation of curve evolution can be easily extended to higher dimensions, and allows computing geometric characteristics of objects in a straightforward manner; (c) there are several applications in medical image analysis where anatomical entities can be enclosed within a closed contour. Level set segmentation consists of evolving a curve to delineate the target object. The curve evolution equations are obtained by optimizing a functional which, generally, contains a data term measuring the conformity of the observed photometric data within each region to a given statistical description. In most of existing region-based level set methods, the data term can be posed following the Maximum Likelihood (ML) principle ${ }^{2}$ [34]-[38]. This corresponds to maximizing the conditional probability of pixel intensity given the assumed model distributions within the objects. As discussed recently by Michailovich et al. [10], Likelihood-based curve evolution is sensitive to inaccuracies in estimating the model distributions. More importantly, it can not incorporate information about the overlap between the distributions of intensity within different regions. Embedding such information in level set segmentation is among the contributions of the current study.

Recent studies have shown the advantages and effectiveness of using distribution metrics in level set segmentation [10], [11] and tracking [12], [13]. Possible measures of similarity/dissimilarity between distributions include the Bhattacharyya coefficient [10], [12] and the mutual information [14]. However, the Bhattacharyya coefficient has shown superior performances over other criteria [10], [12]. In [10], tow-region segmentation is stated as minimizing the similarity (or maximizing the dissimilarity) between the nonparametric ${ }^{3}$ distributions sampled from inside and outside the curve. In [12], [13], the target object is identified as the region whose intensity distribution most closely matches a learned distribution. It has been demonstrated experimentally [12] that curve evolution based on the Bhattacharyya measure outperforms the ML principle. Furthermore, it is much less sensitive to inaccuracies in estimating model distributions [10]. Similar to likelihood-based methods, these studies assume implicitly that the overlap between the distributions of intensity within different regions in the current image/frame has to be minimal. Unfortunately, such assumption is often violated in cardiac imagery as well as many other applications.

\footnotetext{
${ }^{2} \mathrm{~A}$ comprehensive review of variational level-set methods can be found in [34]

${ }^{3}$ In order to incorporate complex statistical information in the segmentation/tracking algorithms, the recent trend in general-purpose segmentation has been toward using nonparametric models [10]-[14].
}

\section{B. The LV tracking/segmentation}

The LV tracking/segmentation is still challenging, although an impressive research effort has been devoted to this task [1]-[9], [15]-[32]. Existing methods are based on, among others, active appearance/shape models [28]-[32], registration [7], clustering [5] and the use of probabilistic atlases via the Expectation-Maximization algorithm [3]. Other studies have addressed specifically the problem of the papillary muscles. A filtering-based approach is investigated in [9] whereas the authors in [8] propose to segment the papillary muscles separately. As several studies have shown [1]-[3], [15][25], the variational framework based on curve evolution has been the most flexible and effective approach to the LV tracking/segmentation. The problem is commonly stated as the optimization of a functional containing a ML intensitydriven constraint and a training-based geometric prior which biases the solution toward geometric knowledge learned from a finite training set [15]-[21]. Although effective in some cases, these methods are subject to the well known limitations of a statistical geometric training: (a) the results depends on the characteristics, variability and mathematical description of a finite training set; (b) a statistical geometric prior can cause systematic bias in some cases [7], particularly with abnormal subjects where the left ventricle undergoes high and unseen (unexpected) geometric variations. Curve coupling between the LV endo- and epicardium was also introduced in [24], [25] in order to prevent the papillary muscle from being included into the myocardium. In [2], the authors propose to maintain a constant photometric environment in the vicinity of the curves propagated from one image to another. This corresponds to matching intensities in profiles perpendicular to the curves in order to maintain their positions constant with respect to anatomical structures. In this connection, we proposed a more global technique in [6] by applying the distribution-matching of intensity [13] to the three cardiac regions: the LV cavity, the myocardium and the background. These recent methods based on intensity matching have led to promising results. However, matching photometric values can not embed information about the overlap between the intensity distributions of different regions. As we will show in this study, embedding global information about such overlap is important and can lead to effective LV tracking.

\section{FORMULATION}

\section{A. The proposed tracking functionals}

Let $\mathcal{I}$ be a MR cardiac sequence containing $N$ frames ${ }^{4}$ $I^{n}: \Omega \subset \mathbb{R}^{2} \rightarrow \mathbb{R}^{+}, n \in[1 . . N]$. The purpose of this study is to automatically detect the endocardium (yellow contour in Fig. 3.a) and the epicardium (green contour in Fig. 3.a) of the heart for each $n \in[2 . . N]$. We formulate the problem as the evolution of two closed planar parametric curves $\vec{\Gamma}_{i n}^{n}(s), \vec{\Gamma}_{\text {out }}^{n}(s):[0,1] \rightarrow \Omega$, toward, respectively, the endo- and epicardium. This consists of segmenting the domain $\Omega$ of each frame into three target regions:

\footnotetext{
${ }^{4}$ The number of frames $N$ is typically equal to 20 or 25 .
} 
(1) The heart cavity $C^{n}$ corresponding to the interior of curve $\vec{\Gamma}_{i n}^{n}$ :

$$
C^{n}=\mathbf{R}_{\vec{\Gamma}_{i n}^{n}}
$$

where $\mathbf{R}_{\vec{\Gamma}}$ denotes the region enclosed by curve $\vec{\Gamma}$ for each $\vec{\Gamma} \in\left\{\vec{\Gamma}_{\text {in }}^{n}, \vec{\Gamma}_{\text {out }}^{n}\right\}$.

(2) The myocardium $M^{n}$ corresponding to the region between $\vec{\Gamma}_{i n}^{n}$ and $\vec{\Gamma}_{\text {out }}^{n}$ :

$$
M^{n}=\mathbf{R}_{\vec{\Gamma}_{i n}^{n}}^{c} \cap \mathbf{R}_{\vec{\Gamma}_{\text {out }}^{n}}
$$

where $\mathbf{R}_{\vec{\Gamma}}^{c}$ denotes the complement of $\mathbf{R}_{\vec{\Gamma}}$ for each $\vec{\Gamma} \in$ $\left\{\vec{\Gamma}_{\text {in }}^{n}, \vec{\Gamma}_{\text {out }}^{n}\right\}$. $\vec{\Gamma}_{\text {out }}^{n}$ :

(3) The background $B^{n}$ corresponding to the region outside

$$
B^{n}=\mathbf{R}_{\vec{\Gamma}_{\text {out }}^{n}}^{c}
$$

For each curve, the evolution equation is obtained by minimizing a cost functional containing three characteristic terms.

\section{(1) Overlap prior terms}

In order to define the proposed overlap priors, we first consider the following definitions:

- For each region $\mathbf{R} \in\left\{C^{n}, M^{n}, B^{n}, n=1 . . N\right\}$, define $P_{\mathbf{R}, I}$ as the nonparametric (kernel-based) estimate of intensity distribution within region $\mathbf{R}$ in frame $I \in\left\{I^{n}, n=1 . . N\right\}$

$$
\forall z \in \mathbb{R}^{+}, P_{\mathbf{R}, I}(z)=\frac{\int_{\mathbf{R}} K(z-I(\mathbf{x})) d \mathbf{x}}{a_{\mathbf{R}}},
$$

where $a_{\mathbf{R}}$ is the area of region $\mathbf{R}$

$$
a_{\mathbf{R}}=\int_{\mathbf{R}} d \mathbf{x}
$$

Typical choices of $K$ are the Dirac function and the Gaussian kernel [10].

- $\mathcal{B}(f / g)$ is the Bhattacharyya coefficient measuring the amount of overlap between two statistical samples $f$ and $g$

$$
\mathcal{B}(f / g)=\sum_{z \in \mathbb{R}^{+}} \sqrt{f(z) g(z)}
$$

Note that the values of $\mathcal{B}$ are always in $[0,1]$, where 0 indicates that there is no overlap, and 1 indicates a perfect match.

We assume that a segmentation of the first frame $I^{1}$, i.e., a partition $\left\{C^{1}, M^{1}, B^{1}\right\}$, is given. Consider

$$
\underbrace{\mathbf{B}_{\text {in }}^{n}=\mathcal{B}\left(P_{C^{n}, I^{n}} / P_{M^{1}, I^{1}}\right)}_{\text {cavity } / \text { myocardium overlap measure }} \quad \forall n \in[1 . . N]
$$

$\mathbf{B}_{i n}^{n}$ measures the amount of overlap between the intensity distribution within the heart cavity region in $I^{n}$ and the myocardium model learned from the first frame. Consider also the following measure of overlap between the intensity distribution within the myocardium region in $I^{n}$ and the background model learned from the first frame:

$$
\underbrace{\mathbf{B}_{\text {out }}^{n}=\mathcal{B}\left(P_{M^{n}, I^{n}} / P_{B^{1}, I^{1}}\right)}_{\text {myocardium/background overlap measure }} \quad \forall n \in[1 . . N]
$$

As we will demonstrate experimentally in section IV, $\mathbf{B}_{i n}^{n}$ and $\mathbf{B}_{\text {out }}^{n}$ are approximately constant over a cardiac sequence.
Consequently, measures $\mathbf{B}_{\text {in }}^{1}$ and $\mathbf{B}_{\text {out }}^{1}$ estimated from a given segmentation of the first frame in sequence $\mathcal{I}$ can be used as overlap priors to constrain the tracking in frames $I^{2} . . I^{N}$. In order to embed prior information about the overlap between the intensity distribution within the three target regions, we propose to minimize the following constraints for each $n \in$ $[2 . . N]$

$$
\begin{gathered}
\mathcal{O}_{\text {in }}^{n}=\underbrace{\left(\mathbf{B}_{\text {in }}^{n}-\mathbf{B}_{\text {in }}^{1}\right)^{2}}_{\text {cavity } / \text { myocardium overlap prior }} \\
\mathcal{O}_{\text {out }}^{n}=\underbrace{\left(\mathbf{B}_{\text {out }}^{n}-\mathbf{B}_{\text {out }}^{1}\right)^{2}}_{\text {myocardium/background overlap prior }}
\end{gathered}
$$

$\mathcal{O}_{i n}^{n}$ measures how the overlap between the intensity distributions within the cavity and myocardium fits prior $\mathbf{B}_{\text {in }}^{1}$. $\mathcal{O}_{\text {out }}^{n}$ measures how the overlap between the intensity distributions within the myocardium and background fits prior $\mathbf{B}_{\text {out }}^{1}$. Although constraining the $\mathrm{LV}$ tracking with overlap priors has an intuitive meaning because of the overlap between the intensity distributions within different cardiac entities (cf. the typical example in Fig. 3), we will further validate experimentally the importance of the proposed overlap constraints (section IV). More precisely, we will confirm with reference (manual) segmentations of 10 datasets, i.e., 190 frames, that $\mathcal{O}_{i n}^{n}$ and $\mathcal{O}_{\text {out }}^{n}$ are approximately equal to zero (refer to table III). Consequently, the manual segmentations correspond approximately to the minima of the overlap priors. Thus, it is expected that, using only the current data, the minimization of such priors would bias the results toward the desired segmentations. As we will show in the experiments, the overlap priors prevent both the papillary muscles from being included erroneously in the myocardium and the curves from spilling into the background, thereby relaxing the need of a geometric training.

Note also that, in the scope of general-purpose segmentation, the overlap priors in (9) can be viewed as a generalization of the segmentation/tracking constraints proposed recently in the studies in [10]-[14]. The latter assume implicitly that the overlap between the distributions within different regions is minimal. The assumption of minimal overlap is often violated in cardiac imagery as well as many other medical applications. The overlap constraint in (9) relaxes such assumption and, consequently, is more applicable ${ }^{5}$ and more versatile than existing intensity-driven constraints-it addresses situations in which an overlap exists between the distributions of different regions.

\section{(2) Mean-matching terms}

In conjunction with the overlap priors, we use meanmatching terms which measure the conformity of intensity means within the cavity and the myocardium in the current

${ }^{5}$ For instance, the particular case corresponding to $\mathbf{B}_{i n}^{1}=0$ is an explicit form of assuming that the overlap between the distributions of the LV cavity and the myocardium is minimal. Such assumption is implicit in existing methods. 
frame to mean priors learned from the first frame

$$
\begin{aligned}
\mathcal{M}_{\text {in }}^{n} & =\underbrace{\left(\mu_{\text {in }}^{n}-\mu_{\text {in }}^{1}\right)^{2}}_{\text {cavity mean prior }} \\
\mathcal{M}_{\text {out }}^{n} & =\underbrace{\left(\mu_{\text {out }}^{n}-\mu_{\text {out }}^{1}\right)^{2}}_{\text {myocardium mean prior }}
\end{aligned}
$$

where $\mu_{i n}^{n}$ and $\mu_{o u t}^{n}$ are the estimates of intensity means within, respectively, $C^{n}$ and $M^{n}$, for $n \in[1 . . N]$

$$
\begin{aligned}
\mu_{i n}^{n} & =\frac{\int_{C^{n}} I^{n} d \mathbf{x}}{a_{C^{n}}} \\
\mu_{\text {out }}^{n} & =\frac{\int_{M^{n}} I^{n} d \mathbf{x}}{a_{M^{n}}}
\end{aligned}
$$

\section{(3) Regularization/gradient terms}

We use classic regularization/gradient terms to bias the curves toward high gradient of intensity and to enforce curve smoothness

$$
\begin{aligned}
\mathcal{G}_{\text {in }}^{n} & =\oint_{\vec{\Gamma}_{\text {in }}^{n}}\left(g_{n}+c\right) d s \\
\mathcal{G}_{\text {out }}^{n} & =\oint_{\vec{\Gamma}_{\text {out }}^{n}}\left(g_{n}+c\right) d s
\end{aligned}
$$

where $c$ is a positive constant and $g_{n}$ is an edge indicator function

$$
g_{n}=\frac{1}{1+\left\|\nabla I^{n}\right\|^{2}} \quad \forall n \in[1 . . N]
$$

The functionals to minimize are a weighted sum of the three characteristic terms

$$
\begin{aligned}
& \mathcal{F}_{i n}^{n}=\alpha \mathcal{O}_{i n}^{n}+\beta \mathcal{M}_{i n}^{n}+\lambda \mathcal{G}_{i n}^{n} \\
& =\underbrace{\alpha\left(\mathbf{B}_{\text {in }}^{n}-\mathbf{B}_{\text {in }}^{1}\right)^{2}}_{\text {cavity/myocardium overlap prior }} \\
& +\underbrace{\beta\left(\mu_{i n}^{n}-\mu_{\text {in }}^{1}\right)^{2}}_{\text {Cavity mean matching }} \\
& +\underbrace{\lambda \oint_{\vec{\Gamma}_{i n}^{n}}\left(g_{n}+c\right) d s}_{\text {Endocardium boundary }} \\
& \mathcal{F}_{\text {out }}^{n}=\alpha \mathcal{O}_{\text {out }}^{n}+\beta \mathcal{M}_{\text {out }}^{n}+\lambda \mathcal{G}_{\text {out }}^{n} \\
& =\underbrace{\alpha\left(\mathbf{B}_{\text {out }}^{n}-\mathbf{B}_{\text {out }}^{1}\right)^{2}}_{\text {myocardium/background overlap prior }} \\
& +\underbrace{\beta\left(\mu_{\text {out }}^{n}-\mu_{\text {out }}^{1}\right)^{2}}_{\text {Myocardium mean matching }} \\
& +\underbrace{\lambda \oint_{\vec{\Gamma}_{\text {out }}^{n}}\left(g_{n}+c\right) d s}_{\text {Epicardium boundary }}
\end{aligned}
$$

\section{B. Minimization equations via curve evolution}

The curve evolution equations are obtained by the EulerLagrange descent minimization of $\mathcal{F}_{\text {in }}^{n}$ and $\mathcal{F}_{\text {out }}^{n}$. To this end, we embed each curve $\vec{\Gamma}, \vec{\Gamma} \in\left\{\vec{\Gamma}_{\text {in }}^{n}, \vec{\Gamma}_{\text {out }}^{n}\right\}$, in a one-parameter family of curves: $\vec{\Gamma}(s, t):[0,1] \times \mathbf{R}^{+} \rightarrow \Omega$, and solve the partial differential equations

$$
\begin{aligned}
\frac{\partial \vec{\Gamma}_{i n}^{n}(s, t)}{\partial t} & =-\frac{\partial \mathcal{F}_{i n}^{n}}{\partial \vec{\Gamma}_{i n}^{n}} \\
\frac{\partial \vec{\Gamma}_{o u t}^{n}(s, t)}{\partial t} & =-\frac{\partial \mathcal{F}_{o u t}^{n}}{\partial \vec{\Gamma}_{o u t}^{n}}
\end{aligned}
$$

where $\frac{\partial \mathcal{F}}{\partial \vec{\Gamma}}$ denotes the functional derivative of $\mathcal{F}$ with respect to $\vec{\Gamma}$. We adopt a two-step algorithm: the first step consists of fixing $\vec{\Gamma}_{\text {out }}$ and evolving $\vec{\Gamma}_{\text {in }}$ (the endocardium boundary) until convergence and the second step evolves $\vec{\Gamma}_{\text {out }}$ (the epicardium boundary) with $\vec{\Gamma}_{i n}$ fixed.

Step 1: In this step, we derive the evolution of $\vec{\Gamma}_{i n}$ by minimizing $\mathcal{F}_{i n}^{n}$. We have

$$
\begin{aligned}
\frac{\partial \vec{\Gamma}_{i n}^{n}(s, t)}{\partial t}=-\frac{\partial \mathcal{F}_{i n}^{n}}{\partial \vec{\Gamma}_{i n}^{n}} & =\underbrace{-2 \alpha\left(\mathbf{B}_{i n}^{n}-\mathbf{B}_{i n}^{1}\right)}_{\text {Overlap prior influence }} \frac{\partial \mathbf{B}_{i n}^{n}}{\partial \vec{\Gamma}_{i n}^{n}} \\
& -2 \beta\left(\mu_{i n}^{n}-\mu_{i n}^{1}\right) \frac{\partial \mu_{i n}^{n}}{\partial \vec{\Gamma}_{i n}^{n}} \\
& -\lambda \frac{\partial \mathcal{G}_{i n}^{n}}{\partial \vec{\Gamma}_{i n}^{n}}
\end{aligned}
$$

Before deriving the final evolution equation of $\vec{\Gamma}_{i n}^{n}$, we give a simple interpretation of how the overlap prior guides the curve evolution. The overlap measure learned from the first frame, $\mathbf{B}_{i n}^{1}$, influences the sign of the multiplicative coefficient (overlap prior influence) affected to the gradient flow $\frac{\partial \mathbf{B}_{i n}^{n}}{\partial \vec{\Gamma}_{i n}^{n}}$. This coefficient is negative when $\mathbf{B}_{i n}^{n}$ is superior to its expected value $\mathbf{B}_{i n}^{1}$. In this case, the overlap prior results in a curve evolution which decreases $\mathbf{B}_{i n}^{n}$. By contrast, when $\mathbf{B}_{i n}^{n}$ is inferior to $\mathbf{B}_{i n}^{1}$, the coefficient becomes positive and the curve evolution increases $\mathbf{B}_{i n}^{n}$. The overlap prior leads to a curve evolution which keeps the overlap between the intensity distributions within the the cavity and the myocardium close to its expected value $\mathbf{B}_{i n}^{1}$. As we will see in the results (c.f. the example in Fig. 3), this prevents the papillary muscles from being included in the myocardium and $\vec{\Gamma}_{i n}^{n}$ from spilling into the background.

To derive the final curve evolution equation, we need to compute $\frac{\partial \mathbf{B}_{i n}^{n}}{\partial \vec{\Gamma}_{i n}^{n}}$. We have

$$
\frac{\partial \mathbf{B}_{i n}^{n}}{\partial \vec{\Gamma}_{i n}^{n}}=\frac{1}{2} \sum_{z \in \mathbb{R}^{+}} \sqrt{\frac{P_{M^{1}, I^{1}}}{P_{C^{n}, I^{n}}}} \frac{\partial P_{C^{n}, I^{n}}}{\partial \vec{\Gamma}_{i n}^{n}}
$$

Now we need to compute $\frac{\partial P_{C^{n}, I}^{n}}{\partial \vec{\Gamma}_{i n}^{n}}$. To this end, we consider the following proposition, which will be used also in the rest of the computation.

Proposition 1: For a scalar function $h$ and a curve $\vec{\Gamma}$, the functional derivative with respect to $\vec{\Gamma}$ of the integral of $h$ over the region enclosed by $\vec{\Gamma}$, i.e., $\mathbf{R}_{\vec{\Gamma}}$, is given by

$$
\frac{\partial \int_{\mathbf{R}_{\vec{\Gamma}}} h(\mathbf{x}) d \mathbf{x}}{\partial \vec{\Gamma}}=h(\mathbf{x}) \vec{n}(\mathbf{x})
$$

where $\vec{n}(\mathbf{x})$ is the outward unit normal to $\vec{\Gamma}$ at $\mathbf{x}$. This result is based on the Green's theorem and has been demonstrated in [41]. 
Applying this proposition to $a_{C^{n}}$ and $\int_{C^{n}} K\left(z-I^{n}(\mathbf{x})\right) d \mathbf{x}$ in $\frac{\partial P_{C n, I^{n}}}{\partial \vec{\Gamma}_{i n}^{n}}$ yields, after some algebraic manipulations

$$
\frac{\partial P_{C^{n}, I^{n}}(z)}{\partial \vec{\Gamma}_{i n}^{n}(s)}=\frac{1}{a_{C^{n}}}\left(K\left(z-I^{n}(s)\right)-P_{C^{n}, I^{n}}(s)\right) \vec{n}_{i n}^{n}(s)
$$

where $\vec{n}_{i n}^{n}$ is the outward unit normal to $\vec{\Gamma}_{i n}^{n}$. We assume $K$ is the Dirac function to simplify the equations. However, the same derivation applies for an arbitrary kernel. Embedding (19) into (17), and after some algebraic manipulations, we obtain:

$$
\frac{\partial \mathbf{B}_{i n}^{n}}{\partial \vec{\Gamma}_{i n}^{n}(s)}=\frac{1}{2 a_{C^{n}}}\left(\sqrt{\frac{P_{M^{1}, I^{1}}(s)}{P_{C^{n}, I^{n}}(s)}}-\mathbf{B}_{i n}^{n}\right) \vec{n}_{i n}^{n}(s)
$$

To compute $\frac{\partial \mu_{i n}^{n}}{\partial \vec{\Gamma}_{i n}^{n}}$, we adopt the same procedure by applying proposition 1 to $a_{C^{n}}$ and $\int_{C^{n}} I^{n}(\mathbf{x}) d \mathbf{x}$ in $\mu_{i n}^{n}$. This yields, after some algebraic manipulations

$$
\frac{\partial \mu_{i n}^{n}}{\partial \vec{\Gamma}_{i n}^{n}}=\frac{I^{n}-\mu_{i n}^{n}}{a_{C^{n}}}
$$

Embedding (20), (21), and the derivative of the regularization/gradient term [39] in (16) gives the final curve evolution equation of the endocardium boundary:

$$
\begin{aligned}
\frac{\partial \vec{\Gamma}_{i n}^{n}}{\partial t} & =\left\{\frac{\alpha\left(\mathbf{B}_{i n}^{n}-\mathbf{B}_{i n}^{1}\right)}{a_{C^{n}}}\left(\mathbf{B}_{i n}^{n}-\sqrt{\frac{P_{M^{1}, I^{1}}}{P_{C^{n}, I^{n}}}}\right)\right. \\
& +\frac{2 \beta\left(\mu_{i n}^{n}-\mu_{i n}^{1}\right)}{a_{C^{n}}}\left(\mu_{i n}^{n}-I^{n}\right) \\
& \left.+\lambda\left[\nabla g_{n} \cdot \vec{n}_{i n}^{n}-\left(g_{n}+c\right) \kappa_{i n}^{n}\right]\right\} \vec{n}_{i n}^{n}
\end{aligned}
$$

where $\kappa_{i n}^{n}$ is the mean curvature function of $\vec{\Gamma}_{i n}^{n}$.

Step 2: In this step we fix $\vec{\Gamma}_{i n}^{n}$ obtained at convergence i.e., when $t \rightarrow \infty$, and evolve $\vec{\Gamma}_{\text {out }}^{n}$ by minimizing $\mathcal{F}_{\text {out }}^{n}$. Following the same computation as in step 1 , and after some algebraic manipulations, we obtain the following evolution equation of the epicardium boundary

$$
\begin{aligned}
\frac{\partial \vec{\Gamma}_{\text {out }}^{n}}{\partial t} & =\left\{\frac{\left(\mathbf{B}_{\text {out }}^{n}-\mathbf{B}_{\text {out }}^{1}\right)}{a_{M^{n}}}\left(\mathbf{B}_{\text {out }}^{n}-\sqrt{\frac{P_{B^{1}, I^{1}}}{P_{M^{n}, I^{n}}}}\right)\right. \\
& +\frac{2 \beta\left(\mu_{\text {out }}^{n}-\mu_{\text {out }}^{1}\right)}{a_{M^{n}}}\left(\mu_{\text {out }}^{n}-I^{n}\right) \\
& \left.+\lambda\left[\nabla g_{n} \cdot \vec{n}_{\text {out }}^{n}-\left(g_{n}+c\right) \kappa_{\text {out }}^{n}\right]\right\} \vec{n}_{\text {out }}^{n}
\end{aligned}
$$

where $\vec{n}_{\text {out }}^{n}$ is the outward unit normal to $\vec{\Gamma}_{\text {out }}^{n}$, and $\kappa_{\text {out }}^{n}$ is the mean curvature function of $\vec{\Gamma}_{\text {out }}^{n}$. Partition $\left(C^{n}, M^{n}, B^{n}\right)$ of frame $I^{n}$ is obtained from $\vec{\Gamma}_{i n}^{n}$ and $\vec{\Gamma}_{\text {out }}^{n}$ at convergence.

\section{Implementation}

1) Level set evolution equations: We use the level-set formalism [42] to implement the curve evolution equations in (22) and (23). We represent curves $\vec{\Gamma}_{i n}^{n}$ and $\vec{\Gamma}_{\text {out }}^{n}$, respectively, by two functions $u_{i n}^{n}: \Omega \subset \mathbb{R} \rightarrow \mathbb{R}\left(\vec{\Gamma}_{i n}^{n}=\left\{u_{i n}^{n}=0\right\}\right)$ and $u_{\text {out }}^{n}: \Omega \subset \mathbb{R} \rightarrow \mathbb{R}\left(\vec{\Gamma}_{\text {out }}=\left\{u_{\text {out }}^{n}=0\right\}\right)$, with the region inside $\vec{\Gamma}\left(\vec{\Gamma}=\vec{\Gamma}_{i n}^{n}, \vec{\Gamma}_{\text {out }}^{n}\right)$ corresponding to $u<0$ $\left(u=u_{i n}^{n}, u_{\text {out }}^{n}\right)$. The level-set representation of curve evolution has well-known advantages over an explicit discretization of $\vec{\Gamma}$
TABLE I

SYNTHETIC EXAMPLE-STATISTICS OF THE BINARY SEGMENTATIONS OBTAINED WITH THREE ENERGIES: DM, DMBM, AND DMOP. THE LEARNED OVERLAP MEASURE IS EQUAL TO $\mathbf{0 . 7 1 .}$

\begin{tabular}{|c|c|c|c|}
\hline Method & DM & DMBM & DMOP \\
\hline Normalized mean at convergence & 0.28 & 0.30 & 0.27 \\
\hline Similarity at convergence & 0.99 & 0.99 & 0.99 \\
\hline Overlap at convergence & 0.63 & 0.42 & 0.71 \\
\hline
\end{tabular}

TABLE II

CARDIAC CAVITY EXAMPLE-STATISTICS OF THE BINARY SEGMENTATIONS OBTAINED WITH THREE ENERGIES: DM, DMBM, AND DMOP. THE LEARNED OVERLAP MEASURE IS EQUAL TO 0.53.

\begin{tabular}{|c|c|c|c|}
\hline Method & DM & DMBM & DMOP \\
\hline Normalized mean at convergence & 0.46 & 0.44 & 0.47 \\
\hline Similarity at convergence & 0.98 & 0.96 & 0.98 \\
\hline Overlap at convergence & 0.50 & 0.44 & 0.54 \\
\hline
\end{tabular}

using a number of points on the curve [42]. It can be effected by stable numerical schemes [42]. It is also implicit, intrinsic and parameter free. The level-set representation of variational problems has become very popular in medical image analysis for several reasons:

- The obtained algorithms can be extended to higher dimensions in a straightforward manner.

- Geometric characteristics of the curve, such as curvatures and normals, can be easily derived.

One can show that [42] if the curve evolves according to $\frac{\partial \vec{\Gamma}}{\partial t}=V \cdot \vec{n}$ (refer to equations (22) and (23)), where $\vec{n}$ is the outward unit normal to $\vec{\Gamma}$ and $V$ a scalar function which depends on pixel position and algorithmic time $t$, then the level set function evolves according to $\frac{\partial u(x, t)}{\partial t}=V \cdot\|\vec{\nabla} u\|$.

2) Weighting parameters: The parameters weighting the relative contribution of the overlap priors, the mean-matching terms and the boundary terms are fixed for all the experiments: $\alpha=1000, \beta=10, \lambda=0.1, c=10$.

3) Data: The algorithm was applied to cine-MR short axis images obtained with a 1.5 Tesla scanner. All the images were obtained during a 10-15 seconds breath-holds with a temporal resolution of 20 or 25 frames. Note that the algorithm is applicable to other types of images.

\section{EXPERIMENTS}

In the following, we first show two-region segmentation examples including a synthetic image and a cardiac image, which demonstrate explicitly the positive effect of the proposed overlap prior and how it can lead to improvements over related distribution-based constraints. Second, we give a typical cardiac example that demonstrates clearly the advantage of using overlap constraints over commonly used ML intensity constraints. Third, we report the statistics of the overlap priors/measures over ten manually segmented datasets, which demonstrates that the overlap measures are approximately constant over a cardiac sequence and, consequently, the minima of the proposed overlap priors correspond approximately to 
TABLE III

STATISTICS OF THE OVERLAP PRIORS/MEASURES (EXPRESSED AS MEAN \pm STD) OVER TEN DATASETS. THE OVERLAP PRIORS ARE approximately EQUAL TO ZERO.

\begin{tabular}{|c|c|c|c|}
\hline $\mathbf{B}_{\text {out }}^{n}$ & $\mathbf{B}_{\text {in }}^{n}$ & $\mathcal{O}_{\text {out }}^{n}=\left(\mathbf{B}_{\text {out }}^{n}-\mathbf{B}_{\text {out }}^{1}\right)^{2},(n>1)$ & $\mathcal{O}_{i n}^{n}=\left(\mathbf{B}_{i n}^{n}-\mathbf{B}_{\text {in }}^{1}\right)^{2},(n>1)$ \\
\hline $0.69 \pm 0.05$ & $0.42 \pm 0.17$ & $1.9\left(10^{-3}\right) \pm 2.4\left(10^{-3}\right)$ & $6.5\left(10^{-3}\right) \pm 8.5\left(10^{-3}\right)$ \\
\hline
\end{tabular}

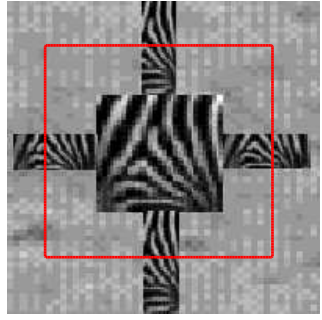

(a) Initialization

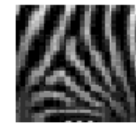

(b) Target object

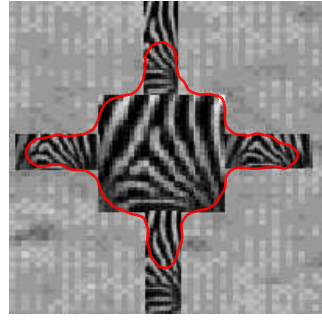

$\mathrm{DM}$

final

curve

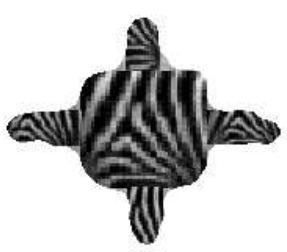

DM

final

object

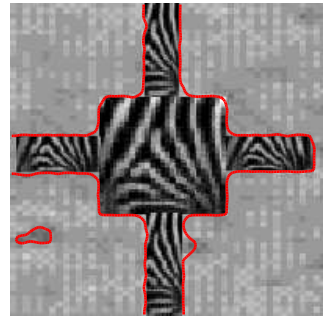

DMBM

final

curve

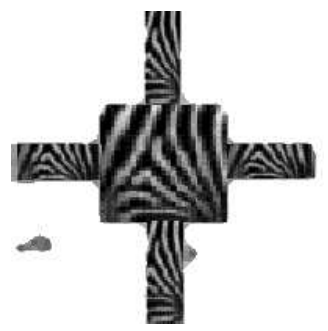

DMBM

final

object

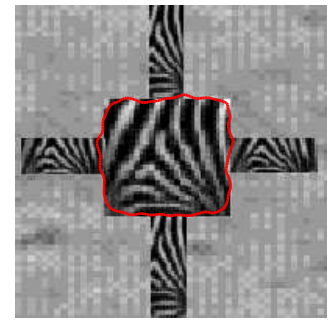

DMOP

final

curve

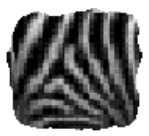

DMOP

final

object

Fig. 1. Segmentation of a synthetic image into two regions with three energies: the second, third, and fourth columns depict the curve at convergence and the obtained object with, respectively, DM, DMBM, and DMOP. Without the overlap prior, i.e. with DM and DMBM, parts of the background were included in the final region. On the contrary, the overlap prior, i.e., DMOP, led to a region similar to the target object in (b). The same initialization in (a) was used for both energies.

reference segmentations. Fourth, we describe a quantitative performance evaluation of the proposed method over ten cardiac datasets by comparisons with manual segmentations and other variational methods [1], [3], [9], [6]. Finally, we give a representative sample of the results for visual inspection.

\section{A. Effect of overlap constraints}

1) Overlap constraints vs. existing distribution-based constraints: To show explicitly the influence of the overlap prior, we compared three energies in the case of segmentation into two regions (an object and a background), one energy contains an overlap prior and the two others do not contain an overlap prior:

(1) DM: The distribution matching energy proposed in [13] and applied to cardiac imagery in [6]. The optimization of this energy seeks a region in the image, so that the sample distribution of the region most closely matches a model distribution.

(2) DMBM: The concatenation of the energy in [13], i.e., $\mathrm{DM}$, and the foreground/background mismatching energy in [10]. Optimization of the latter seeks a segmentation, so that the similarity between the foreground and background distributions is minimal.
(3) DMOP: The concatenation of DM and the proposed overlap prior.

Fig. 1 shows a synthetic example in which the target object is a texture square in the middle of the image. The second and third columns depict the curve at convergence and the obtained object with, respectively, DM and DMBM. With these two energies, i.e., without the overlap prior, parts of the background, which have an intensity profile similar to the target square, were included in the final region. On the contrary, with the proposed overlap prior, i.e., with DMOP, the final curve delineates accurately the square in the middle of the image, leading the a region similar to the target object (refer to the fourth column in Fig. 1). The overlap prior prevents the curve from spilling into the background even with a curve initialization far from the target object (refer to Fig. 1 a). For the three energies, we used the same initialization, smoothness constraint, and model distributions learned from the same image. The cardiac image example in Fig. 2, where the purpose is to separate the heart cavity (refer to the manual segmentation in Fig. 2 b and c) from the background with only intensity information, illustrates the same effect of the proposed overlap prior. It depicts an instance where both DM 


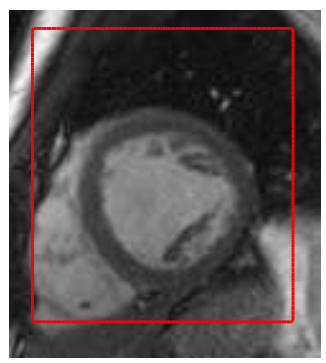

(a) Initialization

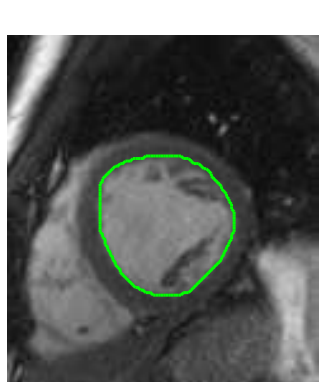

(b) Manual delineation

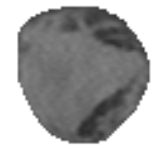

(c) Target object

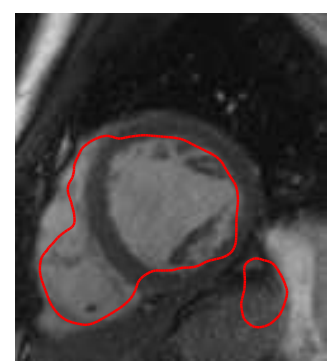

DM

evolution

step

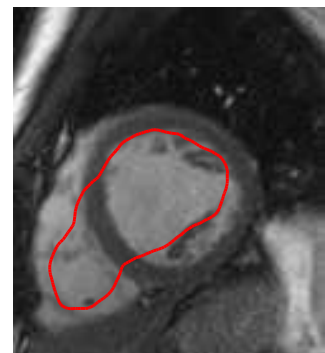

DM

final

curve

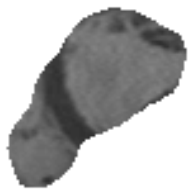

DM

final

object

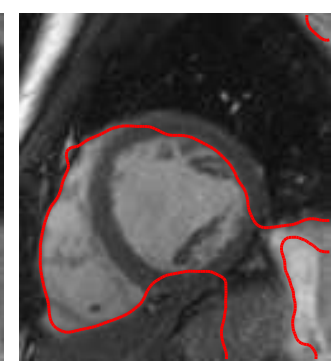

DMBM

evolution

step

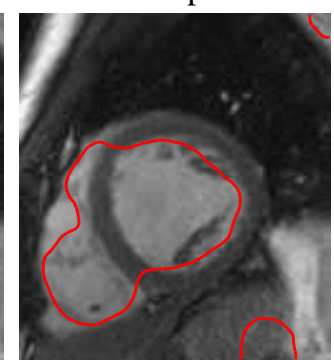

DMBM

final

curve

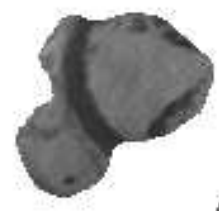

DMBM

final

object

ข

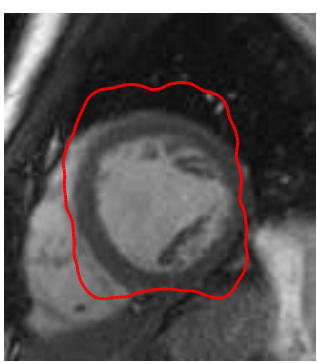

DMOP

evolution

step

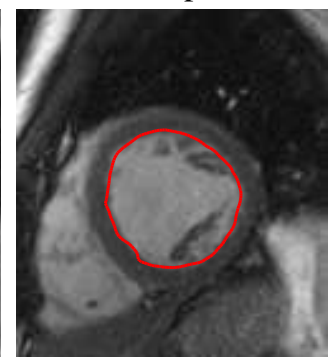

DMOP

final

curve

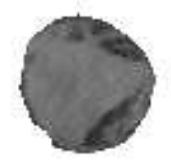

DMOP

final

object

Fig. 2. Segmentation of a cardiac image into two regions with three energies: DM, DMBM, and DMOP. The purpose is to separate the cavity from the background as shown by the manual segmentation in (b) and (c). The second, third, and fourth columns depict the curve at convergence and the obtained object with, respectively, DM, DMBM, and DMOP. Without the overlap prior, i.e. with DM and DMBM, parts of the right ventricle and myocardium were included in the curve at convergence, whereas adding the proposed overlap prior, i.e., using DMOP, biases the solution accurately toward the cavity. For the three energies, we used the same initialization in (a) and the same model distributions learned from a previous frame.

and DMBM include erroneously parts of the right ventricle and myocardium in the curve at convergence (refer to the second and third columns in Fig. 2), whereas adding the proposed overlap prior, i.e., using DMOP, biases the solution accurately toward the target object, the left ventricle cavity in this case (refer to the fourth columns in Fig. 2). For the three energies, we used the same initialization and the same model distributions learned from a previous frame. These examples demonstrate clearly that the overlap prior is the energy that prevents the curve from spilling, thereby relaxing the need of complicated shape priors. To illustrate quantitatively this positive effect of the overlap prior, we report in tables I and II the following statistics of the binary segmentations obtained with DM, DMBM, and DMOP.

- Normalized mean at convergence: the mean of intensity within the curve at convergence.
- Similarity at convergence: The Bhattacharyya measure of similarity between the distribution of intensity within the curve at convergence and a model distribution, i.e., DM at convergence.

- Overlap at convergence: The Bhattacharyya measure of overlap between the foreground (the region inside the curve) and the background (the region outside the curve) at convergence.

For the three energies, we obtained approximately the same mean and the same similarity with the model distribution (refer to the second and third row in table I and table II), although the segmented objects are different (refer to the last row in Fig. 1 and Fig. 2). On the contrary, adding the proposed overlap prior, i.e., using DMOP, led to a measure of overlap different from those obtained with DM and DMBM and approximately equal to the learned prior (refer to the last row in table I and table II). 
When the curve spills in the background with DM and DMBM, the overlap between the foreground and background decreases, deviating from the learned prior. Consequently, matching the distribution inside the curve to a model distribution as in [13], [6] and using a mean matching constraint are not sufficient for these binary segmentation examples. Adding the overlap prior limited the space of possible solutions, and was necessary to obtain the correct segmentations. These statistics confirm explicitly that the overlap term is the constraint that prevents the curve from spilling in the background.

2) Overlap constraints vs. ML intensity-driven constraints: Figure 3 (a) shows a typical example of a MR mid-cavity frame. It depicts the expected segmentations of the LV cavity (region inside the yellow curve) and the epicardial region (region inside the green curve). Figs. $3 \mathrm{~d}$ and e illustrate the significant overlap between the distributions within the three target regions: cavity, myocardium and background. No geometric-training constraints were added for a fair comparison between overlap and ML constraints. For both constraints, model distributions of regions were estimated from the same pesegmented frame. With a ML intensity-driven constraint, parts of the background, which have intensity profiles similar to the cavity and the myocardium, are included inside the final curves (refer to Fig. 3 c). The use of geometric-training constraints in conjunction with ML constraints is inevitable to obtain satisfying results [15]-[23]. By contrast, using an overlap constraint delineates accurately the cavity and the LV (refer to Fig. 3 b), thereby relaxing the need of a training.

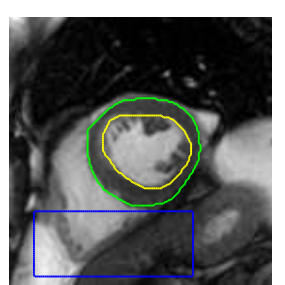

(a) Manual segmentation Overlap LV/Background

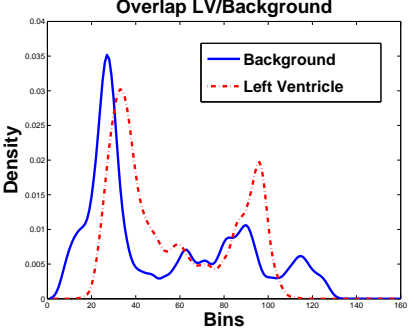

(d)

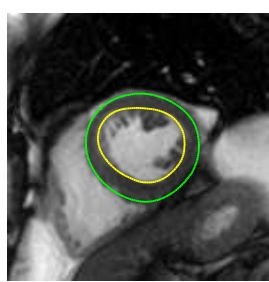

(b) Overlap segmentation

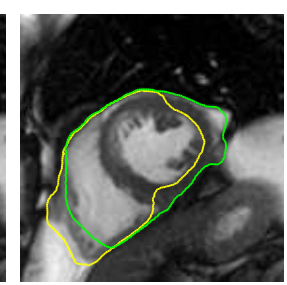

(c) $\mathrm{ML}$

egmentation

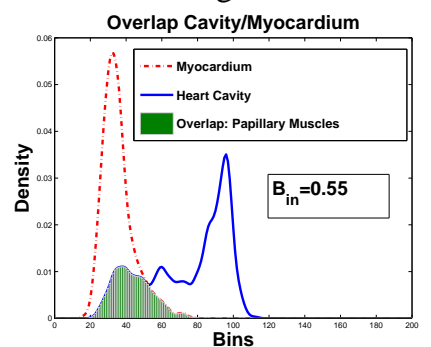

(e)
Fig. 3. Advantage of overlap constraints: (a) manual segmentation by a radiologist-yellow curve: endocardium, green curve: epicardium, region within the yellow curve: cavity, region between the yellow and green curves: myocardium, region outside the green curve: background; (b) segmentations obtained with an overlap constraint, i.e., with our method; (c) segmentations obtained with a ML constraint; (d) overlap between the distributions within the LV and the nearby background (region inside the blue curve in a); (e) overlap between the distributions within the cavity and the myocardium.

\section{B. Statistics of the overlap priors/measures}

In Fig. 4, we plotted the proposed overlap priors/measures versus the frame number (190 frames) using manual segmentations from 10 datasets. As reported in table III, the overlap priors in (9) are approximately equal to zero (refer to the continuous red line in Fig. 4). This validates the usefulness of such priors for the LV tracking. It is also interesting to notice that overlap measures $\mathbf{B}_{i n}^{n}$ and $\mathbf{B}_{\text {out }}^{n}$ do not vary much over different patients (refer to Fig. 4).

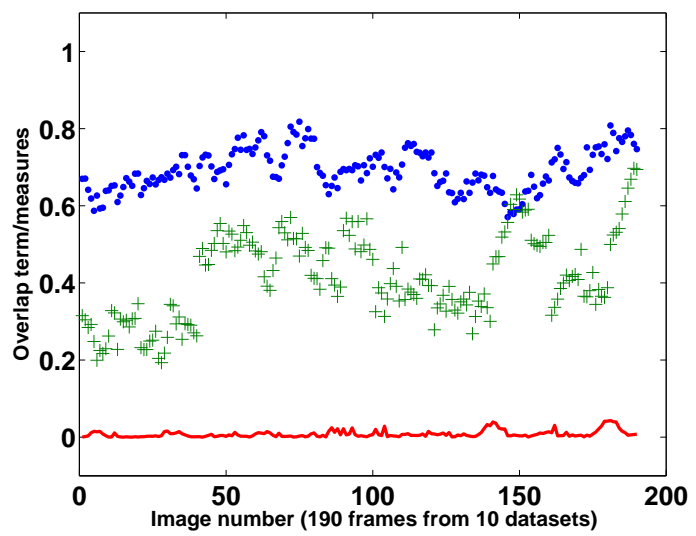

Fig. 4. The proposed overlap priors/measures versus the frame number (190 frames) in 10 manually segmented datasets: $\mathbf{B}_{\text {out }}^{n}$ (blue points); $\mathbf{B}_{i n}^{n}$ (green markers); $\left(\mathbf{B}_{\text {out }}^{n}-\mathbf{B}_{\text {out }}^{1}\right)^{2}+\left(\mathbf{B}_{\text {in }}^{n}-\mathbf{B}_{\text {in }}^{1}\right)^{2}$ (continuous red line).

\section{Quantitative performance evaluation}

The performance of the proposed variational technique was evaluated by comparisons with independent manual segmentations approved by an experienced cardiologist. We applied the method to $2 \mathrm{D}$ mid-cavity MR sequences obtained from 10 subjects, i.e., 10 different datasets: 190 frames were automatically segmented. The free parameters were unchanged for all the datasets: $\alpha=1000, \beta=10, \lambda=0.1, c=10$. Curve initializations and estimations of $\mathbf{B}_{\text {in }}^{1}, \mathbf{B}_{\text {out }}^{1}, \mu_{i n}^{1}$, and $\mu_{\text {out }}^{1}$ were obtained from a user-provided segmentation of the first frame in each sequence. Two clinically important measures were evaluated for performance appraisal: $L V$ cavity area and $L V$ epicardial area. Area measurements are expressed as the number of pixels within the region. We first used the Dice Metric $(D M)$ to measure the similarity between manual and automatic segmentations. Let $\mathbf{A}_{\mathbf{a}}, \mathbf{A}_{\mathbf{m}}$ and $\mathbf{A}_{\mathbf{a} m}$ be the areas of, respectively, the automatically detected region, the corresponding hand-labeled region and the intersection between them. $D M$ is given by ${ }^{6}$ [1]

$$
\frac{2 \mathbf{A}_{\mathbf{a} m}}{\mathbf{A}_{\mathbf{a}}+\mathbf{A}_{\mathbf{m}}}
$$

Our algorithm yielded a $D M$ equal to $0.93 \pm \mathbf{0 . 0 2}$ for all the data (refer to table IV). $D M$ is expressed as mean \pm standard deviation. We obtained $D M$ equal to $0.92 \pm \mathbf{0 . 0 3}$ for the LV cavity areas and $D M$ equal to $0.94 \pm 0.01$ for the epicardial areas.

${ }^{6} D M$ is always in $[0,1] . D M$ equal to 1 indicates a perfect match between manual and automatic segmentation. 
TABLE IV

Dice measures OF SIMILARITY BETWEEN MANUAL AND AUTOMATIC SEgmentations. THE Higher the Dice metric, THE BETTER THE SEGMENTATION.

\begin{tabular}{|c|c|c|}
\hline Dice metrics & Mean & Std \\
\hline This method & 0.93 & 0.02 \\
\hline Method in [1] & 0.81 & 0.16 \\
\hline Method in [6] & 0.89 & 0.09 \\
\hline Method in [9] & 0.90 & 0.05 \\
\hline
\end{tabular}

TABLE V

Correlation coefficients BETWEEN MANUAL AND AUTOMATIC AREAS. THE HIGHER THE correlation coefficient, THE BETTER THE SEGMENTATION.

\begin{tabular}{|c|c|c|}
\hline Correlation & LV cavity areas & Epicardial areas \\
\hline This method & 0.94 & 0.96 \\
\hline Method in [3] & 0.89 & 0.87 \\
\hline Method in [6] & 0.82 & 0.89 \\
\hline
\end{tabular}

We also report good correlation coefficients between manual and automatic endo- and epicardial areas (refer to table V).

To bear comparisons with other recent variational approaches to LV tracking, we give in tables IV and V, respectively, the Dice metrics and correlation coefficients reported in [1], [3], [9], [6]. Although neither a geometric training nor a preprocessing were used, our method achieved a competitive score in comparison with existing variational level set methods. This is consistent with the visual inspection of the results in [1] (cf. the examples given in Fig. 7 in [1]), which show that the method in [1] does not allow embedding midcavity papillary muscles in the inner curve. On the contrary, the proposed method prevents the papillary muscles from being included erroneously in the myocardium (refer to Fig. 5). This is due to the effect of the prior on the overlap between the cavity and the myocardium. It should be noted, however, that the accuracy of the proposed method comes at the price of a user interaction. The method in [1] segments all the slices and is fully unsupervised whereas the proposed method needs contours in the first frame.

\section{Visual inspection}

\section{Mid-cavity slices:}

In Fig. 5, we give a representative sample of the results with five mid-cavity sequences ( $s 1-s 5)$. sxfy depicts the tracking obtained for frame $y$ in sequence $x$. The red and green curves represent, respectively, $\vec{\Gamma}_{i n}^{n}$ and $\vec{\Gamma}_{\text {out }}^{n}$ at convergence. The obtained curves divide each frame into three regions: the heart cavity (region inside the red curve), the myocardium (region between the red and the green curve), and the background (region outside the green curve). Although neither a geometric training [20], [21] nor a preprocessing step [9] were used, the proposed method successfully included the papillary muscles inside the cavity. This task is challenging [9], particularly when the papillary muscles are connected to the myocardium (refer to the typical examples in Fig. 5). Furthermore, unlike existing intensity constraints which do not allow to achieve
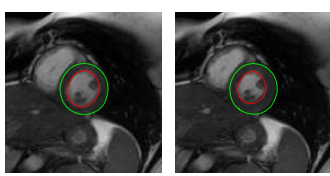

$s 1 f 5$

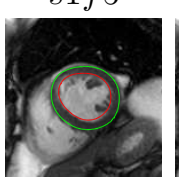

$s 2 f 3$
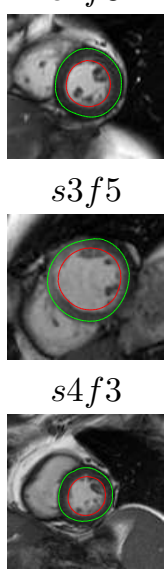

$$
s 5 f 3
$$

$s 1 f 7$

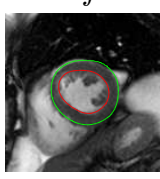

$s 2 f 5$

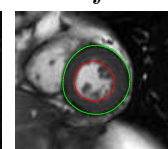

$s 3 f 7$

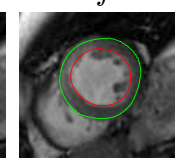

$s 4 f 5$

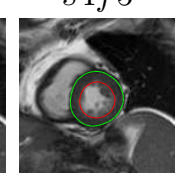

$s 5 f 5$

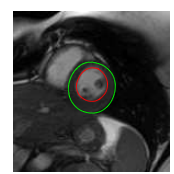

$s 1 f 9$

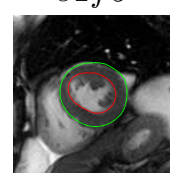

$s 2 f 7$

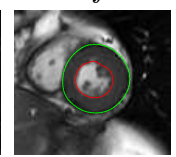

$s 3 f 9$

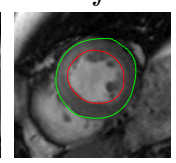

$s 4 f 7$

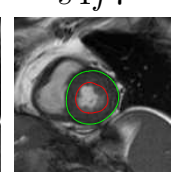

$s 5 f 9$

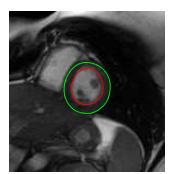

$s 1 f 11$

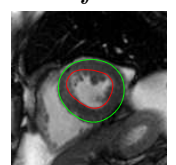

$s 2 f 13$

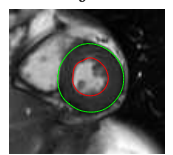

$s 3 f 12$

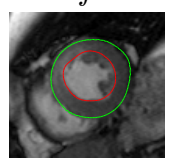

$s 4 f 10$

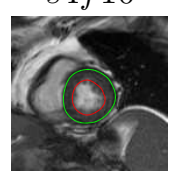

$s 5 f 11$

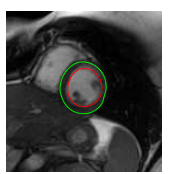

$s 1 f 13$

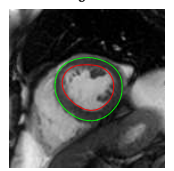

$s 2 f 17$

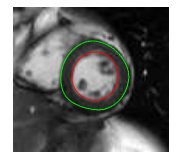

$s 3 f 15$

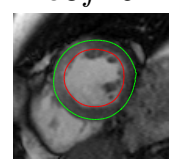

$s 4 f 15$

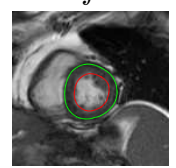

$s 5 f 13$
Fig. 5. Mid-cavity slices-results for five MR sequences: each row depicts the results for one sequence. $\alpha=1000, \beta=10, \lambda=0.1, c=10 . \vec{\Gamma}_{i n}^{n}$ : red curve, $\vec{\Gamma}_{\text {out }}^{n}$ : green curve.

acceptable results without geometric training, the proposed overlap priors prevent both the endo- and epicardium boundaries from spilling into the background.

\section{Basal slices:}

Fig. 6 depicts a representative sample of the results with five basal sequences. These include difficult examples where the slice does not contain the myocardium in all $360^{\circ}$ (cf. $s 1 f 4$ in the first row in Fig. 6). Even with these examples, the proposed method prevented the endo- and epicardium boundaries from spilling into the background, and led to satisfying results.

\section{Apical slices:}

Fig. 7 depicts a representative sample of the results with five apical sequences. These include examples where it is difficult to segment the left ventricle because of the small size of the structures and moving artifacts. Visual inspection of these results demonstrate that the proposed method is flexible and effective, although it uses only the intensity of the current data.

\section{DisCusSION}

This study investigated overlap priors for curve evolution tracking of the LV endo- and epicardium boundaries in cardiac MR sequences. The curve evolution equations were derived by minimizing two functionals each containing an original overlap prior constraint. Using 190 reference (manual) segmentations from 10 datasets, we demonstrated experimentally that the overlap measures are approximately constant over a 


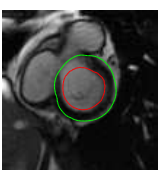

$s 1 f 4$

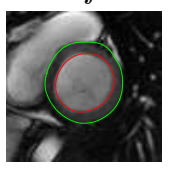

$s 2 f 3$

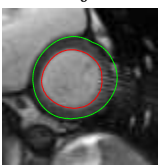

$s 3 f 4$

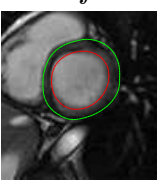

$s 4 f 3$

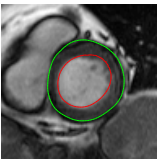

$s 5 f 3$

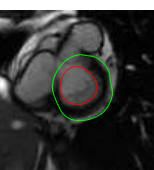

$s 1 f 9$

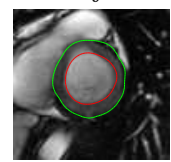

$s 2 f 7$

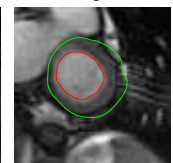

$s 3 f 7$

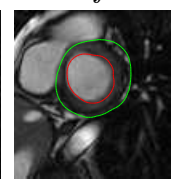

$s 4 f 5$

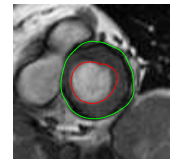

$s 5 f 5$

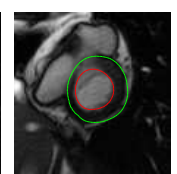

$s 1 f 12$

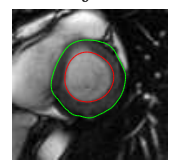

$s 2 f 10$

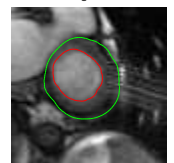

$s 3 f 9$

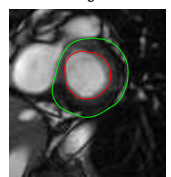

$s 4 f 9$

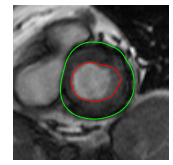

$s 5 f 6$

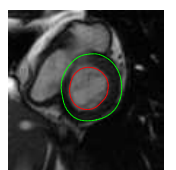

$s 1 f 15$

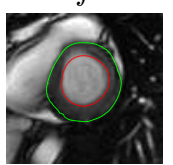

$s 2 f 13$

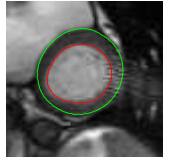

$s 3 f 12$

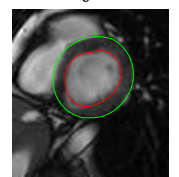

$s 4 f 11$

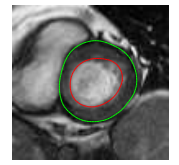

$s 5 f 7$

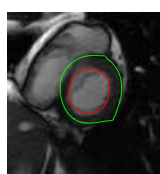

$s 1 f 19$

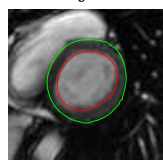

$s 2 f 16$

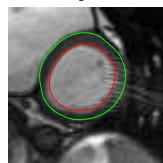

$s 3 f 15$

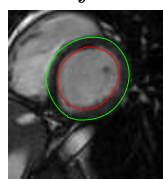

$s 4 f 14$

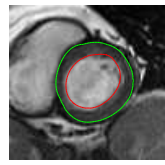

$s 5 f 9$
Fig. 6. Basal slices-results for five MR sequences: each row depicts the results for one sequence. $\alpha=1000, \beta=10, \lambda=0.1, c=10 . \vec{\Gamma}_{i n}^{n}$ : red curve, $\vec{\Gamma}_{\text {out }}^{n}$ : green curve.

cardiac sequence. This led naturally to the proposed overlap energies. Note that a high variation of the overlap in a given sequence will affect segmentation accuracy because the proposed algorithm is based on the assumption that the overlap is approximately constant. Note also that the algorithm was performed on 2D slices. Were a segmentation of the first volume given, it would be straightforward to extend the obtained 2D curve propagation to a 3D surface evolution. The level set representation of evolving interfaces, such as $2 \mathrm{D}$ curves and 3D surfaces, extends to arbitrary higher dimensions. The final evolution equations in (22) and (23) apply also for a surface evolution; the only difference is that the domain of the level set function is $\mathbb{R}^{3}$ rather than $\mathbb{R}^{2}$. However, the $3 \mathrm{D}$ segmentation of the first volume requires either a higher amount of user interaction or an independent automated process.

Although the proposed system uses only the current data, i.e., neither a geometric training nor a preprocessing were used, the final curve evolution equations have several desirable effects:

- The overlap priors prevent the curves from spilling into the background. This has been demonstrated explicitly with two-region segmentation examples. It has been also confirmed by extensive testing over 190 segmentations with 10 different subjects.

- The proposed energies and two-step minimization suffice to prevent the curves from intersecting each other and, therefore, relax the need of explicit curve coupling. This positive

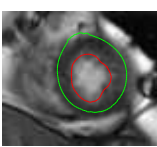

$s 1 f 3$

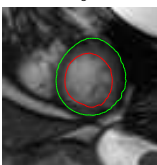

$s 2 f 2$

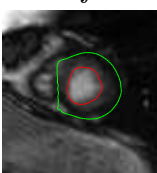

$s 3 f 2$

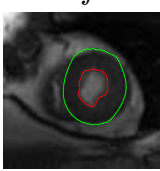

$s 4 f 3$

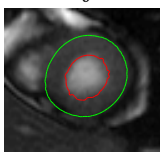

$s 5 f 4$

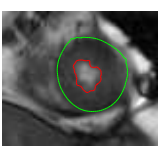

$s 1 f 6$

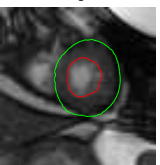

$s 2 f 7$

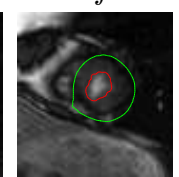

$s 3 f 4$

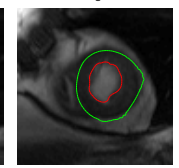

$s 4 f 5$

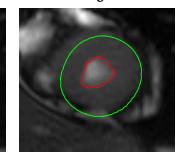

$s 5 f 6$

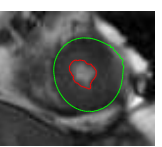

$s 1 f 7$

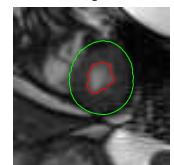

$s 2 f 9$

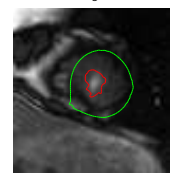

$s 3 f 8$

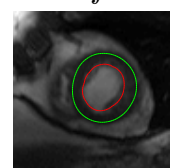

$s 4 f 9$

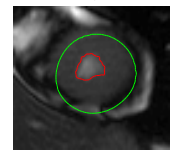

$s 5 f 8$

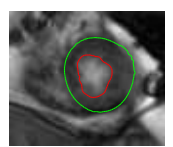

$s 1 f 11$

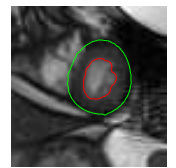

$s 2 f 12$

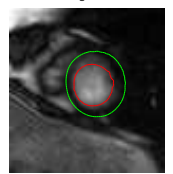

$s 3 f 11$

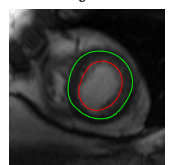

$s 4 f 10$

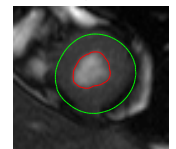

$s 5 f 10$

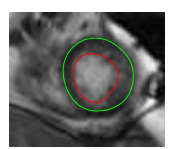

$s 1 f 14$

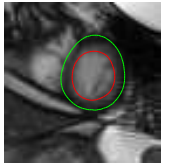

$s 2 f 19$

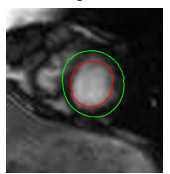

$s 3 f 18$

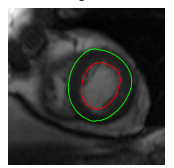

$s 4 f 11$

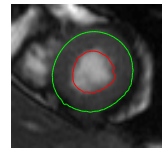

$s 5 f 18$
Fig. 7. Apical slices-results for five MR sequences: each row depicts the results for one sequence. $\alpha=1000, \beta=10, \lambda=0.1, c=10 . \vec{\Gamma}_{i n}^{n}$ : red curve, $\vec{\Gamma}_{\text {out }}^{n}$ : green curve.

effect has been confirmed over the 190 tests we run. In each of these tests, the final curves did not intersect each other. This can be explained by the following effects of the proposed energies:

1) The inner curve cannot spill within the myocardium because this would increase the cavity/myocardium overlap constraint. Therefore, it does not intersect the initial outer curve.

2) When the outer curve propagates within the myocardium erroneously toward the inner curve, we have:

a) The distribution of the region between the two curves-the myocardium region-remains approximately constant because the myocardium has, generally, a homogeneous intensity. Consequently, the myocardium/background overlap constraint does not decrease.

b) The myocardium mean matching constraint has a similar effect; it does not decrease because the mean between the two curves remains approximately constant.

c) On the contrary, the epicardium boundary term increases when the outer curve evolves inward within the myocardium because the myocardium has a homogeneous intensity (the gradient within the myocardium is small).

These positive effects allow more flexibility in clinical use because the results of the proposed method are not bounded to the characteristics, variability, and mathematical description of a finite training set. 


\section{REFERENCES}

[1] M. Lynch, O. Ghita, and P. F. Whelan: Segmentation of the Left Ventricle of the Heart in 3-D+t MRI Data Using an Optimized Nonrigid Temporal Model. IEEE Transactions on Medical Imaging 27(2) (2008) 195-203.

[2] G. Hautvast, S. Lobregt, M. Breeuwer and F. Gerritsen: Automatic Contour Propagation in Cine Cardiac Magnetic Resonance Images. IEEE Transactions on Medical Imaging 25 (9) (2006)

[3] M. Lynch, O. Ghita, and P.F. Whelan. Left-ventricle myocardium segmentation using a coupled level-set with a priori knowledge. Computerized Medical Imaging and Graphics. 30 (2006) 255-262.

[4] M. Lorenzo-Valdés, G. I. Sanchez-Ortiz, A. G. Elkington, R. H. Mohiaddin, and D. Rueckert: Segmentation of 4D cardiac MR images using a probabilistic atlas and the EM algorithm. Medical Image Analysis 8 (2004) 255-265.

[5] M. Lynch, O. Ghita, and PF. Whelam: Automatic segmentation of the left ventricle cavity and myocardium in MRI data. Comput Biol Med. (2006) 36, 389-407

[6] I. Ben Ayed, S. Li, I. Ross and Ali Islam: Myocardium Tracking via Matching Distributions, International Journal of Computer Assisted Radiology and Surgery (2009) 4(1), 37-44.

[7] NMI. Noble, DLG. Hill, M. Breeuwer, J. Schnabel, D. Hawkes, FA. Gerritsen and R. Razavi: Myocardial delineation via registration in a polar coordinate system. Dohi, T., Kikinis, R. (eds.) MICCAI (2002) LNCS, Springer 2488, 651-658.

[8] Spreeuwers, LJ., Bangma, SJ., Meerwaldt, RJHW., Vonken, EJ., Breeuwer, M.: Detection of trabeculae and papillary muscles in cardiac MR images. Computers in Cardiology 32, 415418 (2005)

[9] R. El Berbari, I. Bloch, A. Redheuil, E. D. Angelini, E. Mousseaux, F. Frouin and A. Herment: Automated Segmentation of the Left Ventricle Including Papillary Muscles in Cardiac Magnetic Resonance Images. Proc. of Functional Imaging and Modelling of the Heart (2007) 16113349

[10] O. V. Michailovich, Y. Rathi, and A. Tannenbaum: Image Segmentation Using Active Contours Driven by the Bhattacharyya Gradient Flow. IEEE Transactions on Image Processing. 16(11) (2007) 2787-2801.

[11] T. Georgiou, O. Michailovich, Y. Rathi, J. Malcolm, A. Tannenbaum. Distribution Metrics and Image Segmentation. Linear Algebra and its Applications, 425: 663-672, 2007.

[12] T. Zhang and D. Freedman. Improving performance of distribution tracking through background mismatch. IEEE Transactions on Pattern Analysis and Machine Intelligence. 27(2) (2005) 282-287.

[13] D. Freedman and T. Zhang: Active contours for tracking distributions. IEEE Transactions on Image Processing. 13(4) (2004) 518-526.

[14] J. Kim, J. W. Fisher III, A. Yezzi, M. Cetin, and A. S. Willsky. A nonparametric statistical method for image segmentation using information theory and curve evolution. IEEE Transactions on Image processing, 14(10): 1486-1502, 2005.

[15] K. D. Fritscher, R. Pilgram, and R. Schubert: Automatic cardiac 4-D segmentation using level sets. Proc. of Functional Imaging and Modelling of the Heart, A. Frangi, Ed. : Springer. (2005) 113-122.

[16] M. R. Kausa, J. von Berga, J. Weesea, W. Niessenb and V. Pekar: Automated segmentation of the left ventricle in cardiac MRI. Medical Image Analysis 8(3) (2004) 245-254.

[17] Y. Wang and Y. Jia: Segmentation of the Left Ventricle from MR Images via Snake Models Incorporating Shape Similarities. IEEE International Conference on Image Processing (2006) 213-216.

[18] C. Pluempitiwiriyawej, J.M.F. Moura, Yi-Jen Lin Wu and Chien Ho: STACS: new active contour scheme for cardiac MR image segmentation. IEEE Transactions on Medical Imaging (2005) 24 (5) 593-603.

[19] D. Rueckert and P. Burger: Shape-based segmentation and tracking in 4-D cardiac MR images. Proc. Int. Conf. Computer Vision, Virtual Reality Robotics Medicine, CVRMed., Grenoble, France (1997) 43-52.

[20] T. Kohlberger, D. Cremers, M. Rousson, R. Ramaraj, and G. Funka-Lea: 4D Shape Priors for a Level Set Segmentation of the Left Myocardium in SPECT Sequences. MICCAI (2006), 92-100.

[21] W. Sun, M. Çetin, R. Chan, V. Reddy, G. Holmvang, V. Chandar and A. Willsky: Segmenting and Tracking the Left Ventricle by Learning the Dynamics in Cardiac Images. Information Processing in Medical Imaging (2005) 553-565.

[22] A. Tsai, A. Yezzi, W. Wells, C. Tempany, D. Tucker, A. Fan, W. E. Grimson, and A. Willsky: A shape-based approach to the segmentation of medical imagery using level sets. IEEE Transactions on Medical Imaging 22(2) (2003) 137-154.

[23] N. Paragios: A level set approach for shape-driven segmentation and tracking of the left ventricle. IEEE Transactions on Medical Imaging 22 (2003) 773-776.
[24] L. Spreeuwers and M. Breeuwer: Detection of left ventricular epi- and endocardial borders using coupled active contours. in Proc. CARS (2003), 1147-1152.

[25] N. Paragios: A Variational Approach for the Segmentation of the Left Ventricle in Cardiac Image Analysis. International Journal of Computer Vision 50(3) (2002) 345362.

[26] M. E. Leventon, O. Faugeras, W. E. L. Grimson, and W. M. Wells: Level Set Based Segmentation with Intensity and Curvature Priors, MMBIA 2000.

[27] M-P. Jolly: Automatic Segmentation of the Left Ventricle in Cardiac MR and CT Images. International Journal of Computer Vision 70(2)(2006) $151-163$.

[28] A. Andreopoulos, J. K. Tsotsos: Efficient and Generalizable Statistical Models of Shape and Appearance for Analysis of Cardiac MRI. Medical Image Analysis, 12(3) (2008) 335-357.

[29] S. Zambal, J. Hladůvka and K. Bühler: Improving Segmentation of the Left Ventricle Using a Two-Component Statistical Model. MICCAI (2006) 151-158.

[30] S. C. Mitchell, J. G. Bosch, B. P. Lelieveldt, R. J. van der Geest, J. H. Reiber, M. Sonka: 3-D active appearance models: Segmentation of cardiac MR and ultrasound images. IEEE Transactions on Medical Imaging $\mathbf{2 1}$ (9) (2002) $1167-1178$.

[31] S. C. Mitchell, B. P. F. Lelieveldt, R. J. van der Geest, H. G. Bosch, J. H. C. Reiber, and M. Sonka: Multistage Hybrid Active Appearance Model Matching: Segmentation of Left and Right Ventricles in Cardiac MR Images. IEEE Transactions on Medical Imaging 20(5) (2001) 415423.

[32] M. B. Stegmann, H. Ólafsdóttir, and H. B. W. Larsson: Unsupervised motion-compensation of multi-slice cardiac perfusion MRI. Medical Image Analysis 9(4) (2005) 394-410.

[33] T. Cootes, G. Edwards and C. Taylor: Active Appearance Models. IEEE Transactions on Pattern Analysis and Machine Intelligence. 23(6) (2001) 681-685.

[34] D. Cremers, M. Rousson, and R. Deriche: A Review of Statistical Approaches to Level Set Segmentation: Integrating Color, Texture, Motion and Shape. International Journal of Computer Vision. 62 (2007) 249-265.

[35] I. Ben Ayed, N. Hennane, and A. Mitiche: Unsupervised Variational Image Segmentation/Classification using a Weibull Observation Model. IEEE Transactions on Image Processing. 15(11) (2006) 3431-3439.

[36] T. F. Chan and L. A. Vese. Active Contours without Edges. IEEE Transactions on Image Processing. 10(2) (2001) 266-277.

[37] I. Ben Ayed, A. Mitiche, and Z. Belhadj: Polarimetric Image Segmentation via Maximum-Likelihood Approximation and Efficient Multiphase Level-Sets. IEEE Transactions on Pattern Analysis and Machine Intelligence. 28(9) (2006) 1493-1500.

[38] I. Ben Ayed, A. Mitiche, and Z. Belhadj: Multiregion Level-Set Partitioning of Synthetic Aperture Radar Images. IEEE Transactions on Pattern Analysis and Machine Intelligence. 27(5) (2005) 793-800.

[39] V. Caselles, R. Kimmel, and G. Sapiro: Geodesic active contours. International Journal of Computer Vision. 22(1) (1997) 61-79.

[40] D. Freedman, R. J. Radke, T. Zhang, Y. Jeong, D. M. Lovelock and G. T.Y. Chen: Model-Based Segmentation of Medical Imagery by Matching Distributions. IEEE Transactions on Medical Imaging 24(3) (2005) 281292

[41] S. C. Zhu and A.L Yuille. Region Competition: Unifying Snake/balloon, Region Growing and Bayes/MDL/Energy for multi-band Image Segmentation. IEEE Transactions on Pattern Analysis and Machine Intelligence, 18(9): 884-900, 1996.

[42] J.Sethian. Level Set Methods and Fast Marching Methods. Cambridge University Press (1999).

[43] M. D. Cerqueira, N. J. Weissman, V. Dilsizian, A. K. Jacobs, S. Kaul, W. K. Laskey, D. J. Pennell, J. A. Rumberger, T. Ryan and M. S. Verani. Standardized Myocardial Segmentation and Nomenclature for Tomographic Imaging of the Heart: A Statement for Healthcare Professionals from Cardiac Imaging Committee of the Council on Clinical Cardiology of the American Heart Association. Circulation 105 (2002) 539-542.

[44] B. S. Spottiswoode, X. Zhong, C. H. Lorenz, B. M. Mayosi, E. M. Meintjes and F. H. Epstein. Motion-guided segmentation for cine DENSE MRI. Medical Image Analysis, 13(1) (2009) 105-115. 